\title{
Face aux racines
}

\section{Facing roots}

\section{Ruth Scheps ${ }^{1}$}

${ }^{1}$ docteur en génétique moléculaire (The Weizmann Institute of Science, Rehovot, Israël) ; productrice à France Culture et journaliste à la Radio Suisse Romande jusqu'en 2009 ; rédactrice en chef de la revue Mikhtav Hadash / La Nouvelle Lettre jusqu'en 2019. rscheps@hotmail.com

RÉSUMÉ. Jusqu'au milieu du XXème siècle, les racines végétales ont très peu intéressé les arts et les sciences : tout comme les peintres, les botanistes se focalisaient sur les parties visibles des plantes. Dès le début du XIX ${ }^{\text {ème }}$ siècle cependant (en tout cas en France), les écrivains témoignent d'une réelle fascination pour les racines - plus horrifiée qu'admirative. Dans la première moitié $d u X X^{{ }^{e ̀ m e}}$ siècle, sans doute influencés par la naissance de l'art abstrait, les artistes visuels commencent à les aborder moins comme des formes à apprivoiser que comme des processus à déchiffrer ou à transposer. Certains de ces processus, qui suscitent de nos jours un immense intérêt chez de nombreux botanistes et philosophes, sont examinés ici dans une perspective à la fois scientifique et artistique : l'enracinement; la transition chez un arbre entre l'unicité du tronc et la multiplicité des racines ; l'interconnexion des racines entre elles et avec divers symbiontes. La dernière partie est consacrée aux rapports entre les racines et diverses modalités de la pensée : d'une part les rêves qu'inspirent les racines aux artistes et aux poètes, d'autre part les théories et réflexions qu'elles éveillent de nos jours chez certains scientifiques, écrivains et philosophes.

ABSTRACT. Until the middle of the 20th century, plant roots were of very little interest to the arts and sciences: just like painters, botanists focused on the visible parts of plants. From the beginning of the 19th century, however (at least in France), writers showed a real fascination for roots - more horrified than admiring. In the first half of the 20th century, no doubt influenced by the birth of abstract art, visual artists began to approach them less as forms to be tamed than as processes to be deciphered or transposed. Some of these processes, which nowadays arouse immense interest among many botanists and philosophers, are examined here from both a scientific and an artistic point of view: rooting; the transition in a tree between the unicity of the trunk and the multiplicity of roots; the interconnection of roots among themselves and with various symbionts. The last part is devoted to the relationship between roots and various modalities of thought: on the one hand, the dreams that roots inspire to artists and poets, and on the other hand, the theories and reflections that they arouse today in certain scientists, writers and philosophers.

MOTS-CLÉS. Racines, fascination, ambivalence, obscurité, enchevêtrements, arbres, enracinement, ramification, mycorhize, intelligence végétale, rêve, radicalité.

KEYWORDS. Roots, fascination, ambivalence, darkness, tangle, trees, rooting, branching, mycorrhiza, plant intelligence, dream, radicality.

\section{Introduction}

Les plantes terrestres sont nées il y a 420 millions d'années ou plus ${ }^{1}$. Pendant très longtemps, la botanique et plus généralement, toutes les théories scientifiques sur l'évolution, ont insisté sur la photosynthèse pourvoyeuse d'oxygène atmosphérique, et donc déterminante dans l'apparition de la vie animale. De leur côté, les grands mythes fondateurs de la conscience occidentale, ont surtout traité des fleurs et des arbres porteurs de fruits, au moins depuis le récit biblique du jardin d'Éden. Quant aux artistes inspirés par les plantes, jusqu'au milieu du $\mathrm{XX}^{\text {ème }}$ siècle, ils en ont essentiellement représenté les parties visibles. Souterraines, les racines restaient doublement dans l'ombre, et étaient vues au mieux comme des quasi objets, mystérieux et figés. Les peintres ne les représentaient presque jamais en tant que telles mais en connexion avec le tronc, et les botanistes se contentaient d'en décrire les formes et fonctionnements directement observables.

\footnotetext{
${ }^{1}$ L'apparition des premières plantes terrestres daterait de - 420 MA selon les archives fossilifères, mais peut-être de - 500 MA si l'on se fonde sur les horloges moléculaires. 
Cependant, nombre d'écrivains des $\mathrm{XIX}^{\text {ème }}$ et $\mathrm{XX}^{\text {ème }}$ siècles ont témoigné d'une réelle fascination pour les racines, qui n'est sans doute pas étrangère à leur complexité, leur étrangeté et leur ambivalence. La main ou l'esprit qui voudrait s'en saisir en totalité, se heurte à leurs structures et fonctions complexes (innombrables ramifications et échanges nutritifs) autant qu'à leur diversité (elles sont souterraines, superficielles ou aériennes ; pivotantes, fasciculées, adventives, etc.). Généralement invisibles, ternes et d'aspect nu, elles constituent pour le philosophe Emanuele Coccia «les formes les plus énigmatiques du monde végétal $»^{2}$. Elles incarnent une ambivalence dérangeante vis-à-vis de la vie et de la mort : bien qu'étant l'organe le plus vital de la plante (elles la nourrissent et la stabilisent), leur aspect souvent noueux voire pétrifié évoque plutôt la mort. La sidération causée par cette contradiction vivante, s'exprimera par des voies elles aussi contrastées : les artistes et les écrivains se tourneront vers l'aspect mortifère des racines, les botanistes, vers leurs manifestations vitales.

En partant du premier saisissement issu de l'observation spontanée des racines, nous tenterons de nous frayer un chemin à travers les multiples approches dont elles ont fait l'objet, de la botanique à la philosophie en passant par les arts visuels et la littérature. Au fil de notre cheminement, nous ferons état de confrontations de plus en plus intimes : entre les racines et la terre, qui se nourrissent mutuellement; entre les racines et le tronc des arbres à leur collet; entre les racines et leurs symbiontes (mycorhizes et bactéries). Jusqu'aux rêves fusionnels des artistes et des poètes qui se sont vus devenir racines, et jusqu'aux pensées radicales qu'elles ont inspiré à certains philosophes.

\section{Fascinations}

\subsection{Les racines et le mal}

À partir de la seconde moitié du $\mathrm{XIX}^{\mathrm{ème}}$ siècle et jusqu'à la fin du XX $\mathrm{X}^{\mathrm{ème}}$, l'Europe connaît un engouement littéraire sans précédent pour les racines - sur le mode négatif. Pour la plupart des écrivains et des philosophes de cette époque, les racines ont partie liée avec tous les visages du mal : la contradiction, l'obscurité, la monstruosité, le malheur, la peur, le vice ou la mort.

Hippolyte Taine note ainsi : «Les racines tordues s'accrochent entre les cailloux comme des pieds désespérés [...] $»^{3}$ et Victor Hugo - «Et la racine affreuse et pareille aux serpents / Fait dans l'obscurité de sombres guet-apens ${ }^{4} »-$ ajoute à la laideur visible, la noirceur des intentions, dans une personnification dramatique dont usera également Valéry, un demi-siècle plus tard. Jean-Paul Sartre associe également la racine au serpent, pour sa relation prédatrice à la terre, qui relève de "hiérarchies enchevêtrées" 5 " : «Le vrai mangeur de terre, le serpent le plus terrestre de tous, c'est la racine. [...] La racine mange la terre, la terre mange la racine ${ }^{6}$. » Une formulation que l'on peut rapprocher du constat lapidaire de Nietzsche : « La nourriture du serpent, la terre ${ }^{7} ! »$.

\footnotetext{
2 Emanuele Coccia, La vie des plantes. Une métaphysique du mélange, Paris, éditions Payot \& Rivage, " Bibliothèque Rivages ", 2016, p. 99

${ }^{3}$ Hippolyte Taine, Voyage en Italie, vol. 2. 1866. $5^{\text {ème }}$ édition en 1884 à Paris, Librairie Hachette et $C^{\text {ie }}$

${ }^{4}$ Victor Hugo, Dieu. La fin de Satan, Paris, Nelson, 1914, p. 80. Première édition, 1891 (création dès 1855)

${ }^{5}$ Dans Douglas Hofstadter, Gödel, Escher, Bach : les brins d'une guirlande éternelle, 1999 en anglais ; traduction française, Paris, Dunod, 2000

${ }^{6}$ Jean-Paul Sartre, L'Être et le Néant. Essai d'ontologie phénoménologique, Paris, Gallimard, première parution en 1943, p. 673. Nouvelle édition Gallimard, collection Tel ( $\left.n^{\circ} 1\right)$, corrigée par Arlette Elkaïm-Sartre en 1994, suivie d'un index, 1976

${ }^{7}$ Friedrich Nietzsche, Le Gai Savoir, trad. Henri Albert, Paris, Mercure de France, 1901, p. 19
} 
Comment ne pas citer ici ce fameux passage de La Nausée, dans lequel Sartre, d'abord abattu et effrayé par sa contemplation hallucinée d'une racine, accède soudain à une nouvelle compréhension existentielle ? L'existence comme simple être-là matériel et excessif, inexplicable et en-deçà de toute individualisation : "J'étais tout à l'heure au Jardin public. La racine du marronnier s'enfonçait dans la terre, juste au-dessous de mon banc. Je ne me rappelais plus que c'était une racine. [...] J'étais assis, un peu voûté, la tête basse, seul en face de cette masse noire et noueuse, entièrement brute et qui me faisait peur. [...] Et puis voilà : tout d'un coup, c'était là, c'était clair comme le jour : l'existence s'était soudain dévoilée. [...] Cette racine, avec sa couleur, sa forme, son mouvement figé, était... au-dessous de toute explication. Chacune de ses qualités lui échappait un peu, coulait hors d'elle, se solidifiait à demi, devenait presque une chose ; chacune était de trop dans la racine, et la souche tout entière me donnait à présent l'impression de rouler un peu hors d'elle-même, de se nier, de se perdre dans un étrange excès ${ }^{8}$. $\gg$ Ce tropisme littéraire pour la face obscure des racines est encore confirmé par le poète François Solesmes : «Déraciné, l'arbre livre l'envers du tapis végétal, voile jeté sur les lourds secrets de famille, les forfaits domestiques ; sur les monstres intimes : / pris en flagrant délit d'étreintes et de chevauchements, et de violence indéfinie, un amas de racines reptiles, saillant de rotules raidies et de muscles tétanisés, / dresse le répertoire de la torsion et du ressaut, de la capture et du contournement, et de toute figure et procédure d'extorsion des aveux. / Dans l'expansion d'un sauve-qui-peut, un délire ligneux d'anastomoses et de nodosités ; obscène, un spasme inextricable ${ }^{9}$. $\gg$ Violemment dénudées par leur déracinement, les racines exhibent ici leurs formes tourmentées comme autant de pulsions coupables et folles.

Dans son essai La terre et les rêveries du repos (écrit en forme de contrepoint à La Terre et les rêveries de la volonté), Gaston Bachelard consacre tout un chapitre à « La Racine » (chapitre IX, qui fait suite à celui sur «Le Serpent»). Il remarque d'emblée que ses «valeurs dramatiques se condensent dans cette seule contradiction : la racine est le mort vivant ${ }^{10} \gg:$ morte en apparence, mais éminemment vivante dans son fonctionnement. Une dissonance cognitive que surmonte la botanique exclusivement tournée vers la richesse des processus racinaires. Bachelard lui-même ne s'est d'ailleurs pas arrêté aux aspects négatifs des racines dont les paradoxes nourrissent son imagination : «La racine est toujours une découverte. On la rêve plus qu'on ne la voit. Elle étonne quand on la découvre : n'est-elle pas roc et chevelure, filament flexible et bois dur ? Avec elle, on a un exemple de contradictions dans les choses ${ }^{11}$.»

Enfin nous ne pouvions conclure ce tour d'horizon sans citer le Dialogue de l'Arbre ${ }^{12}$ de Paul Valéry - texte merveilleusement poétique dont le passage suivant suffirait presque à résumer les formes et les forces de toute la dramaturgie racinaire : "Un fleuve tout vivant de qui les sources plongent dans la masse obscure de la terre les chemins de leur soif mystérieuse. C'est une hydre, ô Tityre, aux prises avec la roche, et qui croît et se divise pour l'étreindre ; qui de plus en plus fine, mue par l'humide, s'échevèle pour boire la moindre présence de l'eau imprégnant la nuit massive où se dissolvent toutes choses qui vécurent. Il n'est bête hideuse de la mer plus avide et plus multiple

\footnotetext{
${ }^{8}$ Jean-Paul Sartre, La Nausée, Paris, Gallimard, première publication, 1938. Coll. « Folio », 2014. pp. 180-181

${ }^{9}$ François Solesmes, Éloge de l'arbre, Paris, Encre marine, 1995, pp. 16-17

${ }^{10}$ Gaston Bachelard, La Terre et les rêveries du repos, chap. IX, « La Racine », pp. 290-322. Paris, Librairie José Corti, 1948 , p. 290. Rééd. 1982

${ }^{11}$ Ibid., p. 292

12 Paul Valéry a écrit Dialogue de l'Arbre en 1943, sous l'Occupation. Le 25 octobre 1943, il présente lui-même ce poème en prose rythmée, écrit en référence aux Bucoliques de Virgile, comme une «fantaisie en forme de dialogue pastoral... Des discours, plus ou moins poétiques, consacrés à la gloire d'un Arbre, s'échangent entre un Tityre et un Lucrèce, dont j'ai pris les noms sans les consulter. "
} 
que cette touffe de racines, aveuglément certaines de progrès vers la profondeur et les humeurs de la terre. Mais cet avancement procède, irrésistible, avec une lenteur qui le fait implacable comme le temps. Dans l'empire des morts, des taupes et des vers, l'œuvre de l'arbre insère les puissances d'une étrange volonté souterraine ${ }^{13}$. $\gg$ C'est une volonté schopenhauerienne que le poète de l'idée convoque ici, un vouloir-vivre inconscient et premier d'où découle le monde entier car : «Tout aspire et s'efforce à l'existence, et si possible à l'existence organique, c'est-à-dire la vie, et, une fois éclose, à son plus grand essor possible ${ }^{14}$.»

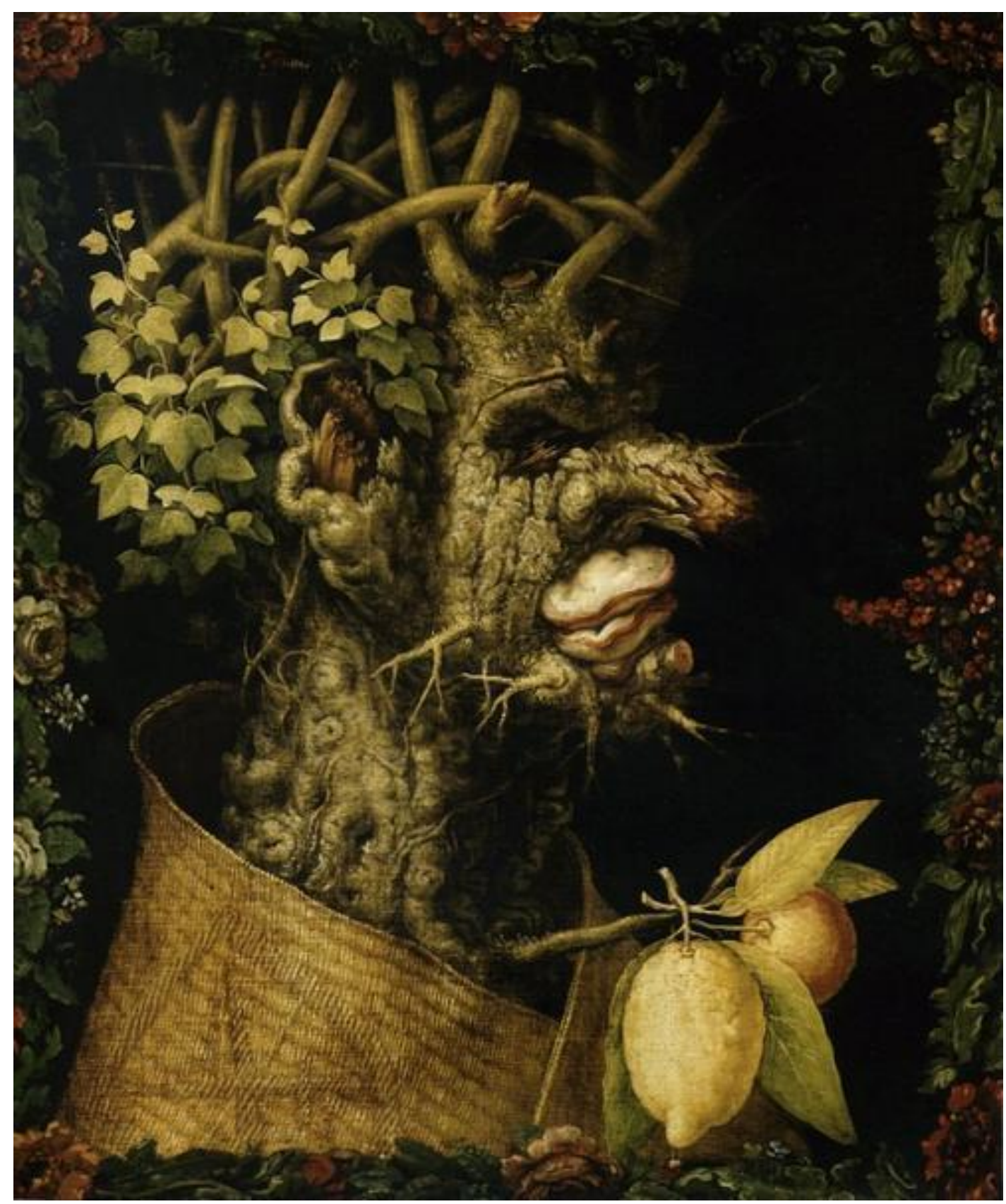

Figure 1. Arcimboldo, L'Hiver, 1573. Huile sur toile, 76 x 63,6 cm Musée du Louvre, Paris

\footnotetext{
13 Paul Valéry, "Dialogue de l'Arbre », dans Eupalinos; L'âme et la danse ; Dialogue de l'Arbre, Paris, NRF Poésie / Gallimard, 1944. Rééd. 2008, pp. 160-161

${ }^{14}$ Volker Spierling, Arthur Schopenhauer, un abécédaire, Paris, éd. du Rocher, 2003. Extrait : Le monde comme volonté et comme représentation, chap. XXVIII, 339, p. 284
} 
Parallèlement à toutes ces manifestations littéraires et négatives de la fascination pour les racines, voyons à présent comment celle-ci s'est répercutée dans l'histoire de la peinture occidentale. Disons d'emblée que rares sont les grands peintres d'arbres inspirés par les racines en tant que telles. C'est l'arbre tout entier qui les intéressait, ou plutôt sa structure aérienne immédiatement visible, à l'exclusion des parties souterraines. Tout au plus celles-ci étaient-elles données à deviner par quelques départs racinaires issus du collet. Ce constat admet toutefois certaines exceptions remarquables, à commencer par le célèbre tableau d'Arcimboldo ${ }^{15}$ intitulé Hiver (Fig. 1), un des quatre portraits composés de sa série allégorique des Quatre saisons ${ }^{16}$. C'est la tête (vue de profil) d'un vieillard ridé dont le visage et le cou sont formés par une vieille souche d'arbre, les cheveux par des branches décharnées et entremêlées sur lesquelles pousse du lierre, et la barbe par des racines chétives et clairsemées. Seuls deux citrons (rappel de l'Italie où ils mûrissent en hiver) ornent le plastron et apportent quelque lumière à ce portrait olivâtre et boursouflé. Il faudra attendre encore un siècle pour voir poindre chez quelques peintres un début d'intérêt envers les racines ${ }^{17}$. Même au XVIII ${ }^{\text {ème }}$ siècle, l'arbre n'a qu'une fonction ornementale dans la peinture de paysages, et ses racines sont totalement absentes. Seules importent sa forme globale et sa place au sein du tableau, qui reste marginale. Vers la fin du $\mathrm{XIX}^{\text {ème }}$ siècle, les peintres, qui suivent de près les écrivains, commencent à considérer les racines comme dignes d'intérêt en elles-mêmes. En témoigne notamment le dernier tableau de Vincent Van Gogh (1853 - 1890), une toile inachevée intitulée Racines $^{18}$ (Fig. 2), peinte le 27 juillet 1890, jour de la mort de Van Gogh (il aurait travaillé toute la journée devant ces racines avant de se suicider). Dans une lettre adressée à son frère Théo en 1882, évoquant deux de ses travaux similaires de l'époque, Vincent écrivait: "Je voulais exprimer, tant dans cette figure de femme blême et mince que dans ces racines noires et bougonnes avec leurs nœuds, quelque chose de la lutte pour la vie. » Il est significatif qu'au seuil d'une mort choisie, Van Gogh ait mis ainsi toute son énergie vitale dans la figuration de ce combat ultime et torturé entre la vie (les frondaisons vertes) et la mort (les racines noueuses aux couleurs cadavériques et plus ou moins confondues avec les troncs et les branchages). Malgré la portée métaphorique de ce tableau, c'est encore de réalisme qu'il s'agit ici : le peintre expressionniste représente à la fois le paysage qu'il a sous les yeux, et son propre paysage intérieur.

\footnotetext{
${ }^{15}$ Arcimboldo (1526 - 1593) : peintre italien qui connut la gloire à la cour des Habsbourg. En 1562, invité à Vienne à la cour de l'empereur Ferdinand I er par son fils Maximilien, il y composa ses fameuses têtes composées - dont Hiver (1573) - tout en organisant les divertissements de la cour. Tombé dans l'oubli après sa mort, il fut redécouvert au XX ${ }^{\text {ème }}$ siècle par les Surréalistes.

${ }^{16}$ La série des Quatre saisons est en correspondance symbolique avec la puissance du souverain (par la diversité des végétaux représentés) et avec la série des quatre éléments, l'Hiver étant lié à l'eau par la figure mythologique de Proserpine (divinité de la mythologie romaine : reine des Enfers et déesse de l'hiver durant les six mois qu'elle y passe sous terre, par ailleurs épouse de Neptune, le dieu de l'Eau.

${ }^{17}$ Par exemple une estampe du peintre néerlandais Karel Dujardin (1626 - 1678), Les arbres à racines découvertes (visible au musée du Louvre), dans laquelle les racines des arbres au premier plan semblent mener leur vie exploratoire de manière quasiment autonome; un dessin du peintre de paysages et graveur italien Crescienzo Onofri (1632 - 1698 ou 1712 ), Paysage arboré, où les racines des deux arbres principaux sont explicitement dans la continuité des troncs qu'elles prolongent.

${ }^{18}$ Racines de Vincent Van Gogh : le lieu précis où ce tableau a été peint - une petite route à quelques pas de la mairie d'Auverssur-Oise - a été récemment découvert, et dévoilé au public à l'occasion des 130 ans de la disparition de l'artiste, suscitant une grande effervescence chez les historiens d'art.
} 


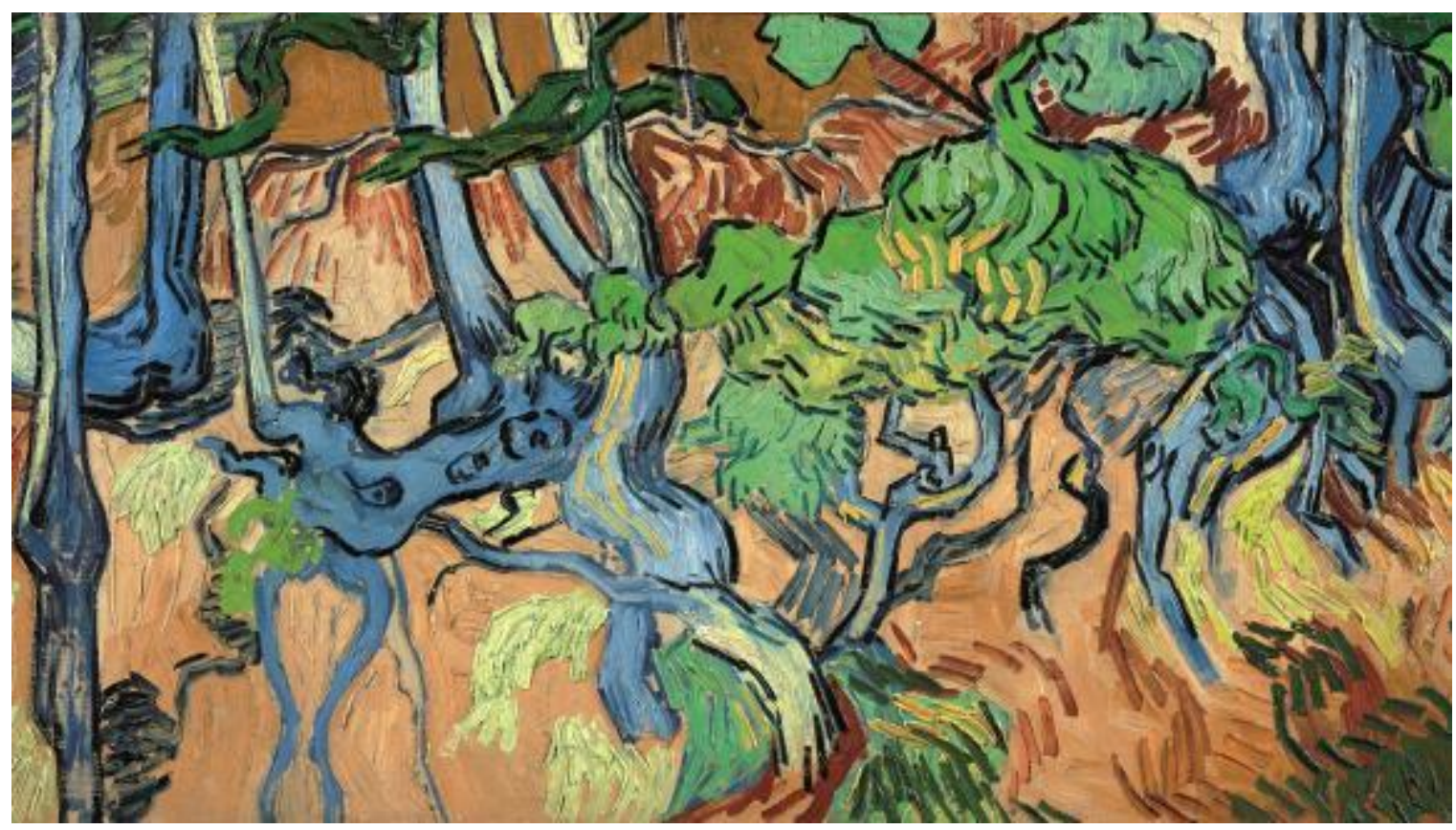

Figure 2. Vincent Van Gogh, Racines, 27 juillet 1890, huile sur toile, 50,3 cm x 100,1 cm. Licence Creative Commons CCO, (C) Musée Van Gogh, Amsterdam (Fondation Van Gogh)

\section{2. Énigmes racinaires}

Dans la première moitié du $\mathrm{XX}^{\mathrm{ème}}$ siècle, la naissance de l'art abstrait influence également les peintres inspirés par la nature. Libérés de la contrainte que représentait l'imitation du réel, ils se donnent d'autres objectifs, plus intérieurs : pénétrer les rouages intimes des choses et les secrets inavouables des êtres - leurs fonctionnements voire leurs rêves. Il ne s'agit plus désormais de montrer le visible tel qu'il est ou qu'il apparaît, mais d'en dévoiler la part la plus profonde.

Dès lors, les artistes visuels abordent les racines moins comme des formes à apprivoiser que comme des processus à déchiffrer voire à transposer. Chez Pierre Alechinsky ${ }^{19}$, la racine devient même la clé de son processus créateur. Hélène Cixous l'affirme d'entrée de jeu dans Le voyage de la racine alechinsky : "Alechinsky entre en art par la racine »- en l'occurrence un rhizome de bambou que le peintre garde dans un tiroir -, « un vieux bout de racine conservée dans une boîte pleine de temps depuis la haute époque de ses premiers commencements. J'en ai le souffle coupé. La racine dort. Vieille. Brunâtre. Ridée. Dessiccée. Je regarde la racine. Elle respire. On dirait son père. On dirait ma mère ${ }^{20}$. » De cette racine originaire, coupée et ravinée mais vivante dans ses circonvolutions, Hélène Cixous fait le cordon ombilical jamais oublié à partir duquel se déploie la dynamique formelle d'Alechinsky. Plus que toute étude scientifique, cette évocation sidérée confère à la racine apparemment momifiée et vivante, une aura ancestrale.

\footnotetext{
19 Pierre Alechinsky (né en 1927) : peintre et graveur belge. Après sa rencontre avec le poète Christian Dotremont, il crée le mouvement Cobra dont le slogan est : "L'imagination au pouvoir ». Son œuvre mêle l'expressionisme au surréalisme. Le thème de l'arbre y est récurrent. Une image centrale est souvent entourée, sur un ou plusieurs côtés, d'une série de vignettes complétant le sens du tableau. Son œuvre intitulée Racines (encre de Chine rehaussée à l'aquarelle sur papier vergé, 318 x 476 $\mathrm{mm}$ ), avec deux grandes vignettes et trois plus petites, présente ainsi cinq variantes de " l'en-mouvement des êtres " (Hélène Cixous) qui renvoient à la quasi animalité des racines.
}

${ }^{20}$ Hélène Cixous, Le voyage de la racine alechinsky, Paris, éditions Galilée, 2012 


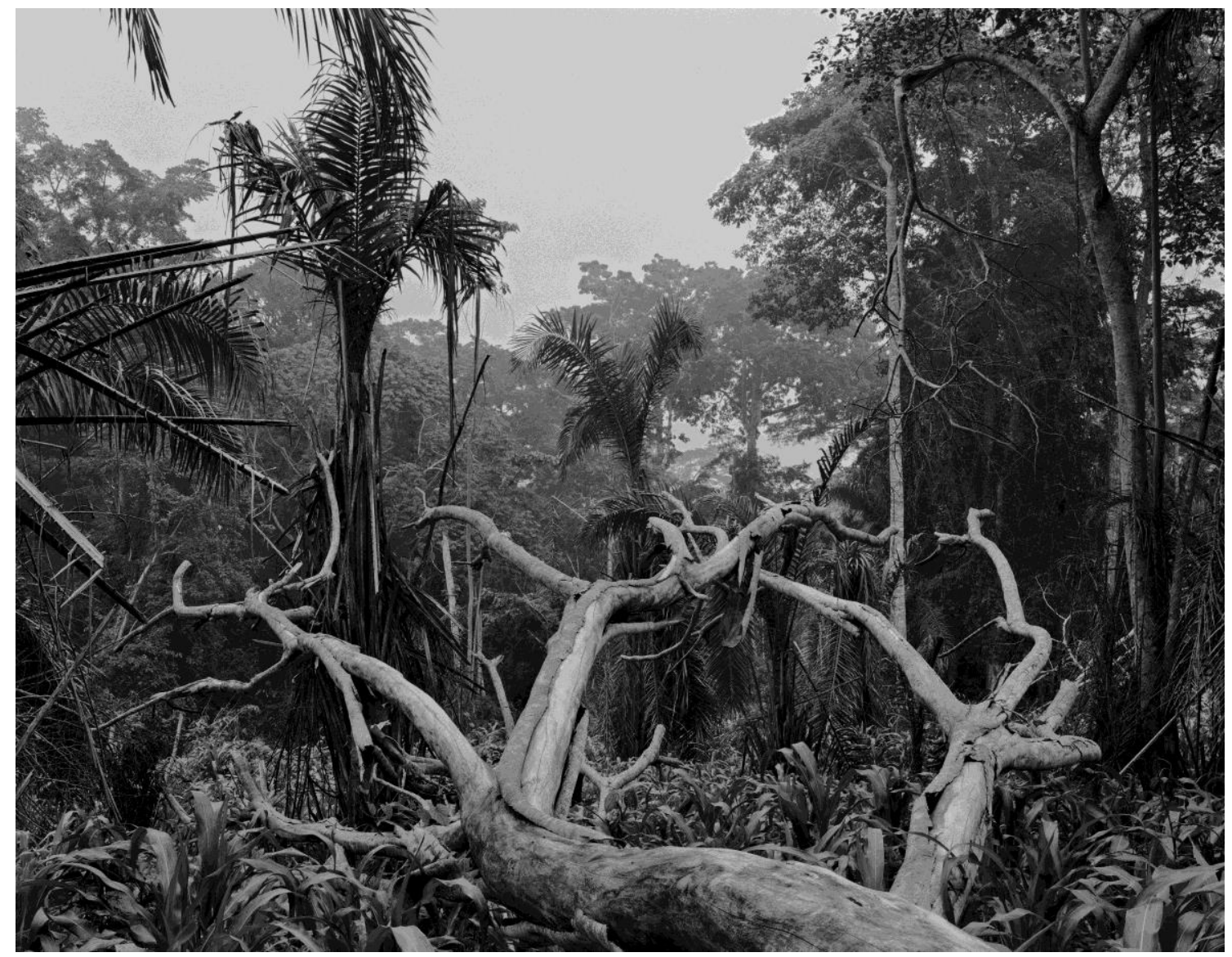

Figure 3. Éric Guglielmi, série «PARADIS PERDU », Cameroun, Lomié, Biba II, juin 2018. Tirage platine Palladium. (C) Courtesy Galerie Maubert-Eric Guglielmi

Il arrive aussi, dans certaines œuvres littéraires ou visuelles, que des éléments végétaux semblent hésiter sur leur propre statut. Le texte «Images terre à terre » du critique d'art Georges DidiHuberman, met en scène des racines qui n'en sont peut-être plus : « Ici (à Rio de Janeiro) on ne va pas à la racine : ce sont les racines qui deviennent lianes dansantes, troncs excentriques, branches innombrables, ramures obsidionales, et qui viennent à nous, courent de partout, nous prennent dans leurs nœuds rythmiques ${ }^{21}$.» Certaines œuvres visuelles contemporaines (dessins, peintures, photographies, sculptures) donnent lieu à des interrogations similaires : le photographe Éric Guglielmi $^{22}$, dans Cameroun Lomié Biba II (Fig. 3), présente un ensemble de ramifications issues d'un tronc mort, témoignage désolé de déforestation intensive - branchages que l'imaginaire pourrait fantasmer en racines lignifiées. Dans sa série des «Enchevêtrements » (Fig. 4), la céramiste Claire Lindner ${ }^{23}$, s'inspirant de racines noueuses observées en 2016 dans les forêts canadiennes, a

\footnotetext{
${ }^{21}$ Georges Didi-Huberman, "Images terre à terre », La besogne des images, sous la direction de Léa Bismuth et Mathilde Girard, co-production Filigranes, Labanque-Béthune, 2019. Non paginé.

22 Éric Guglielmi : photographe et éditeur français, né en 1970 à Charleville-Mézières (Ardennes). Vit et travaille à Paris, représenté par la galerie Maubert. Les photographies issues de ses nombreux voyages relèvent du documentaire engagé. Éric Guglielmi donne à voir les incohérences et les angles morts de réalités géopolitiques qu'il connaît bien. Les êtres et les objets ainsi photographiés, par-delà leur beauté propre, font mémoire des violences humaines qui les ont atteints. Publications : Touba, voyage au coeur d'un islam Nègre, éd. Alternatives, 2007 ; Je suis un piéton rien de plus, éd. Gang, 2011 (sur les traces de Rimbaud) ; What Happens, éd. Gang, 2015 (sur les problématiques des accords transfrontaliers).

${ }^{23}$ Claire Lindner : céramiste française née en 1982 à Perpignan (Pyrénées-Orientales). Depuis 2006, participe à des expositions collectives, notamment la "Scène française contemporaine » au Musée des Arts décoratifs de Paris (2011) et " Le Banquet » au Grand Palais (2015), dans le cadre du Salon Révélations. En 2013, représente la France à la " Triennale européenne de la Céramique et du Verre » du WCC-BF de Mons, en Belgique. Ce même lieu la choisit en 2016 pour le commissariat d'exposition (C) 2020 ISTE OpenScience - Published by ISTE Ltd. London, UK - openscience.fr Page $\mid 7$
} 
assemblé des boudins de céramique qui s'enchevêtrent et se soutiennent délicatement. Le regard qui les parcourt ne peut qu'hésiter : sont-ils en croissance ou en train de se replier ? Et d'ailleurs, s'agitil de racines attirées par la terre, de tiges alanguies ou bien de tentacules, voire de doigts ? Auquel cas on serait en présence d'une œuvre à la fois minérale (sa matière), végétale (son inspiration) et animale (son attribution). Toutes ces représentations artistiques font écho aux ambiguïtés botaniques des racines : rhizomes souterrains, mais qui sont des tiges et non des racines ; racines aériennes comme des tiges; racines d'arbres, dites longues ou de structure, qui se lignifient comme des branches, tandis que d'autres, dites fines, ont plusieurs caractères rappelant ceux des feuilles ${ }^{24}$.

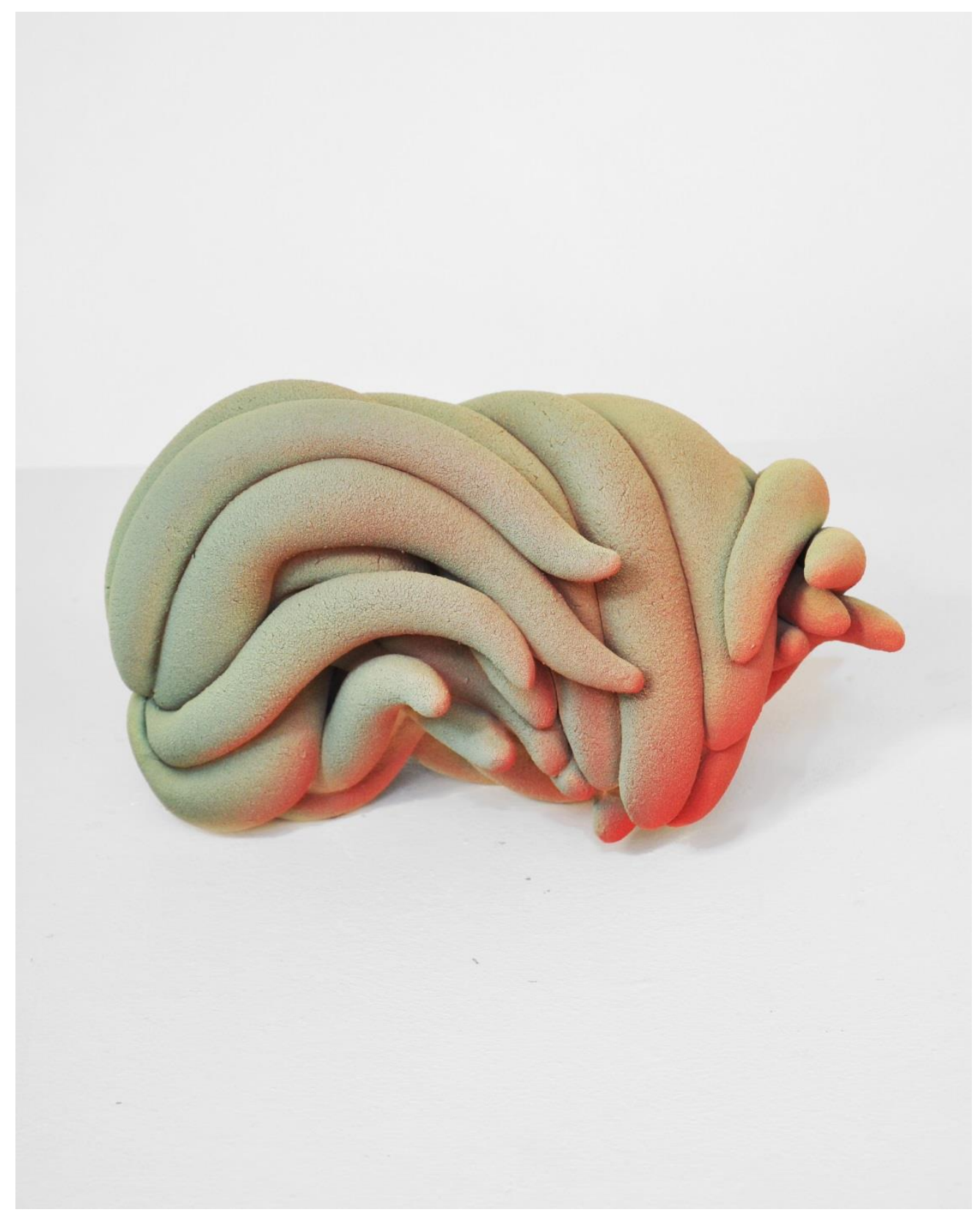

Figure 4. Claire Lindner, série "Enchevêtrements », Enchevêtrement $n^{\circ}$ 5, 2017, Grès chamotté émaillé, $20 \times 31 \times 23 \mathrm{~cm}$. Collection particulière.

sur le thème du « Nuage » - en résonance avec sa première exposition personnelle à la Galerie de l'Ancienne Poste en 2014, intitulée « Measuring Clouds ».

${ }^{24}$ Francis Hallé, «Des feuilles souterraines ? ", Alliage $n^{\circ}$ 64, mars 2009, pp. 90-95 


\section{Explorations}

\subsection{L'enracinement}

La présence de racines n'est pas toujours nécessaire à la vie végétale : certaines plantes aquatiques, épiphytes ou saprophytes n'en possèdent pas du tout, et certaines mousses ne se fixent que par des poils fins et courts, les rhizoïdes. Par ailleurs rappelons que les organes souterrains d'une plante ne sont pas toujours des racines : chez certains végétaux, les organes souterrains sont des tiges ${ }^{25}$. Dans la grande majorité des cas cependant, ce sont de vraies racines qui poussent, attirées par la terre, l'humidité et l'obscurité. Le géotropisme positif caractérise toutes les racines souterraines ainsi que de nombreuses racines aériennes, qui poussent donc de la plante vers le sol. Une radicule se forme, qui constituera la racine principale, puis des racines secondaires, qui prendront plus ou moins d'importance selon les systèmes racinaires. Cependant, comme Darwin l'avait déjà remarqué : «Le géotropisme [...] détermine l'incurvation de la radicule vers le bas ; mais cette force, très peu considérable, est tout à fait insuffisante pour perforer la terre. Cette pénétration s'effectue parce que l'extrémité appointée (protégée par la pilorhize ${ }^{26}$ ) est pressée vers le bas, par suite de l'expansion longitudinale, ou de la croissance de la portion terminale rigide ; cette dernière se trouve encore aidée par la croissance transversale, et l'action cumulative de ces deux forces est considérable ${ }^{27}$. » Loin de subir passivement la gravité terrestre, les plantes déploient donc des moyens considérables pour s'enraciner de plus en plus profondément. Elles croissent ainsi en inventant leurs propres chemins, et sont sensibles ${ }^{28}$ aux obstacles avant même d'être à leur contact, ce qui leur permet de les contourner ; par ailleurs elles forment des systèmes racinaires qui évoluent différemment selon la qualité du sol et la nature des plantes voisines.

Dans la nature, si les conditions sont favorables, une plante vivante s'enracine (activement) ; dans le cas contraire, elle est déracinée (passivement). L'enracinement d'une plante est donc caractérisé par l'accroissement de sa substance racinaire et par suite, de son rayon d'action souterrain. Pour la philosophe Florence Burgat, «son enracinement se produit en un lieu qu'elle ne détermine pas à partir d'elle-même et dont elle ne peut s'échapper ${ }^{29}$ » mais cette affirmation est à nuancer car, bien que la plante ne puisse jamais quitter son lieu d'origine, les extrémités de ses racines ne cessent de s'en éloigner du fait de leur croissance indéfinie, et parfois de phénomènes de réitération à partir de tiges, de branches ou de racines. Un dessin de cep de vigne ancienne par l'artiste Raffi Kaiser ${ }^{30}$ (Fig. 5) montre ainsi (sur la droite) une branche du cep en train de s'enraciner.

\footnotetext{
${ }^{25}$ Tiges souterraines : il en existe de plusieurs types : le drageon, rejet naissant sur racines; le tubercule, organe de réserve à l'extrémité d'une tige ou d'un entre-nœuds ; le rhizome, tige parfois tubérisée, garnie de racines adventives ; le bulbe, organe de réserve formé de plusieurs couronnes de gaines foliaires charnues entourant une très courte tige qui émet quelques racines adventives.

${ }^{26}$ Pilorhize, ou coiffe : capuchon qui protège les tissus embryonnaires du méristème apical

${ }^{27}$ Charles Darwin, La faculté motrice dans les plantes (trad. de l'anglais, annotations et préface par le Dr. Édouard Heckel), Paris, Reinwald, 1882, p. 200

${ }^{28}$ Sensibilité (biologique) : propriété (d'un être vivant, d'un organe) de réagir d'une façon adéquate aux modifications du milieu

${ }^{29}$ Florence Burgat, Qu'est-ce qu'une plante ? Essai sur la vie végétale, Paris, Seuil, 2020, p. 103

${ }^{30}$ Raffi Kaiser : artiste franco-israélien né en 1931 à Jérusalem. A étudié à Jérusalem, à Paris et à Florence. Après une retraite de trois ans dans les déserts d'Israël (Judée et Néguev, 1978), il passe de la peinture de sujets imaginaires et colorés, au dessin de paysages naturels en noir et blanc. En 1987, il fait un séjour de six mois en Chine ; les dessins qu'il en rapporte (Suite chinoise) seront exposés au musée Guimet (1990). En 1991, il passe un an au Japon (montagnes et Kyoto), voyage qui donnera lieu à une deuxième série de dessins (Suite japonaise) et à plusieurs expositions au Japon. Entre 1995 et 1997, il retourne dans le désert du 


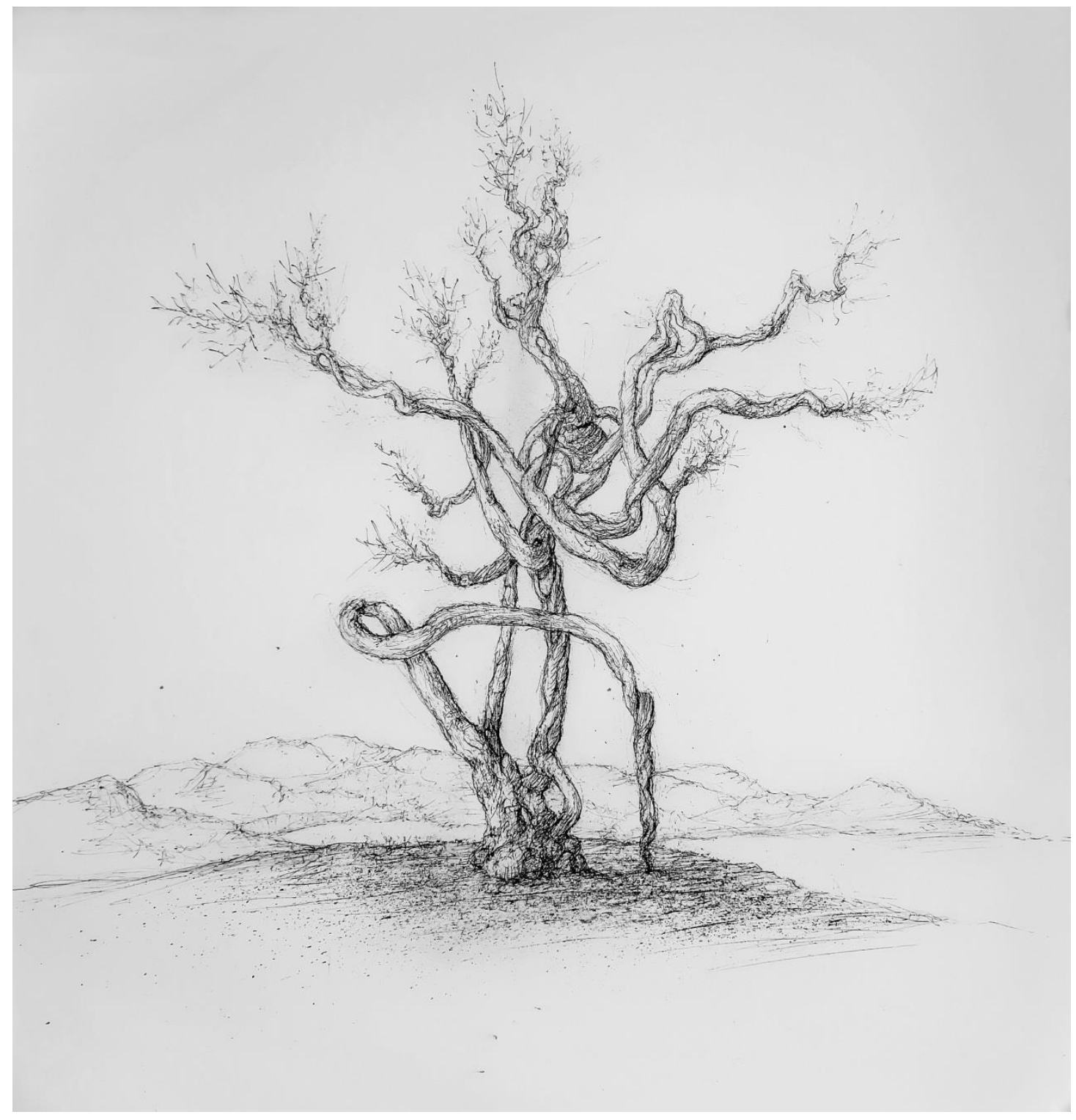

Figure 5. Raffi Kaiser, Sans titre (Toscane), encre et plume, 76 × $56 \mathrm{~cm}$, 2006. Photographie Francis Wasserman. Collection de l'artiste (C) Raffi Kaiser

\subsection{Entre tronc et racines}

Les racines entretiennent avec la tige ou le tronc des rapports diversifiés : la plupart des racines croissent sous terre, en une structure globalement symétrique de l'ensemble aérien de la plante. Mais certaines racines, elles-mêmes aériennes, reviennent auprès du tronc de l'arbre support qu'elles enserrent et étouffent, comme dans le cas emblématique du figuier étrangleur (voir le dessin de Francis Hallé en Fig. 6). 


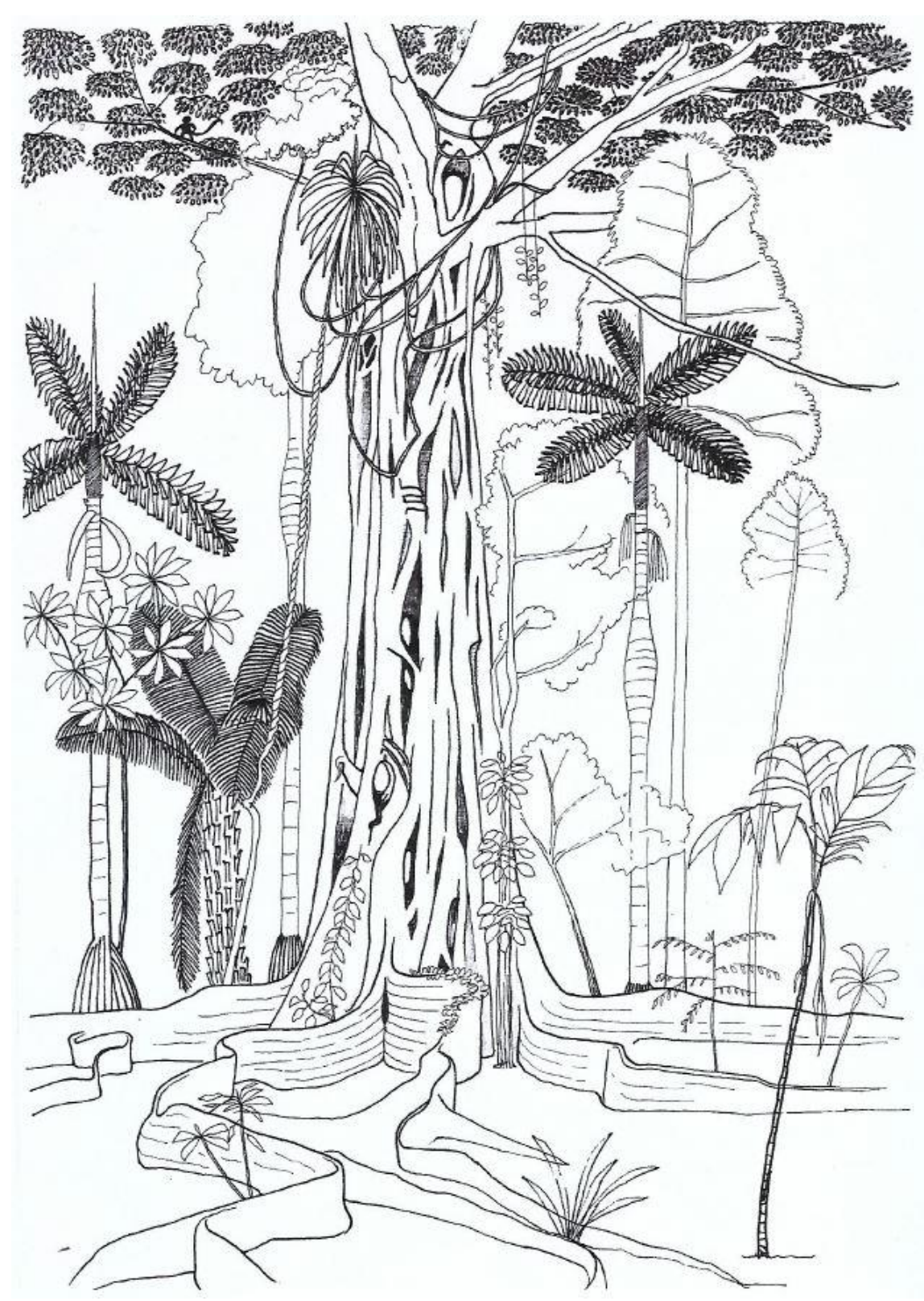

Figure 6. Francis Hallé, Ficus étrangleur, forêt de Pakitza, Amazonie péruvienne, 2012. Crayon et encre sur papier, $42 \times 30 \mathrm{~cm}$. Collection de l'artiste @Francis Hallé

Dans un article paru en 2014, le philosophe Dov Bernard Hercenberg questionne la nature des rapports qu'entretiennent tronc et racines au niveau du collet d'un arbre à cernes. Entreprise difficile s'il en est, car la transition qui s'opère dans le collet entre le tronc et les racines, est invisible : tout se joue à l'intérieur de la matière ligneuse, elle-même complexe en raison des nombreuses racines imbriquées les unes dans les autres. Pour tenter de suivre le passage de l'unité d'un fût (texture à cernes mono-centrée) à la pluralité de ses racines (chacune dotée de son propre tissu à cernes), on a débité en tranches le collet d'un épicéa à plusieurs fûts ${ }^{31}$. Dans la Figure 7 (photographie d'une tranche de bois de $5 \mathrm{~cm}$ ), des motifs circulaires et ovoïdes témoignent de processus d'individuations racinaires : cinq ou six côté fûts (ici), une dizaine côté terre. Chez cet arbre à cernes, le rapport de l'unique (le tronc) au multiple (les racines ou les branches) est particulier car la structure des cernes reste partout la même. Dans une vision descendante, on s'intéressera au passage entre le motif unique des cernes du tronc préexistant, et ce même motif, répété à l'identique dans toutes les racines émergentes. Dans une vision ascendante au contraire, on

\footnotetext{
${ }^{31}$ Travail de Ben Barman au Val Ferret (Valais, Suisse)
} 
prêtera attention à ce qui gouverne l'enracinement des racines en tant que tel, c'est-à-dire leur manière tâtonnante et insistante de se frayer leur propre chemin. Les réflexions de l'auteur sur le collet en tant que lieu de passage et espace partagé, sont inspirées en partie par la méditation heideggérienne sur la vie comme volonté d'indépendance autant que coexistence. Comment le motif des cernes peut-il « à la fois se ramifier, varier dans ses dimensions, se réorienter et conserver sa spécificité structurelle ${ }^{32}$ ? Nous ne pouvons, dans le cadre de ce travail, faire droit à tous les développements philosophiques mobilisés par Hercenberg à partir du collet de l'arbre, et aux réflexions qu'il lui inspire en tant que cas particulier d'une réalité complexe et de mise à l'épreuve de nos possibilités cognitives. À ce propos, l'auteur met le doigt sur la situation paradoxale où nous place l'observation du collet, que nous avons l'impression de pouvoir saisir en totalité, alors même que tout ce qui fait son être-ensemble est invisible car intérieur. De là, il en appelle à un dépassement de l'expérience, motivé par le fait que le savoir, venant aussi de la culture dont nous héritons, est plus vaste et antérieur à l'expérience sensible que nous tirons de nos perceptions.

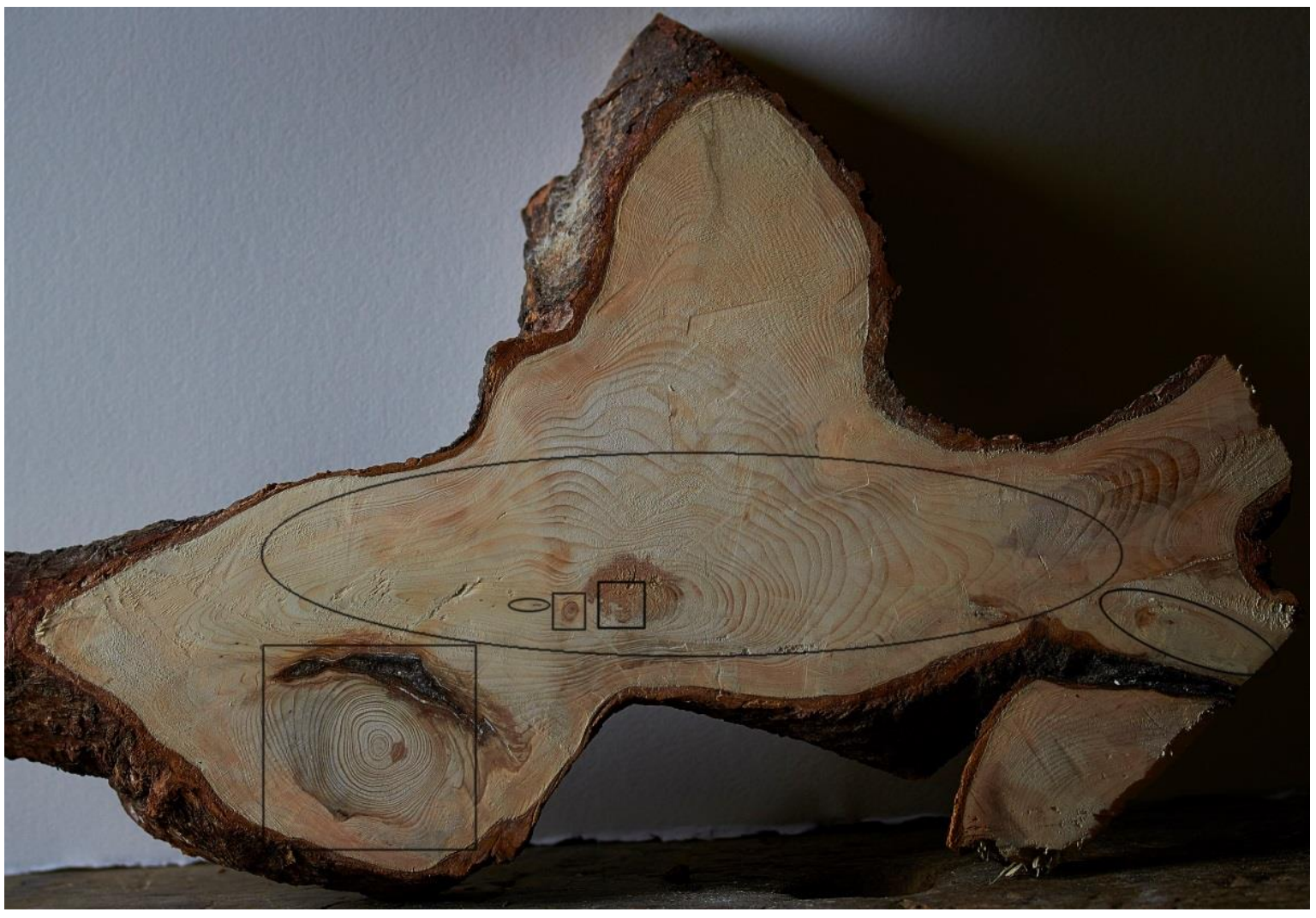

Figure 7. Ben Barman (tronçonnage et polissage) et Dov Bernard Hercenberg (photographie), Suivi des passages côté tronc, 2012

\subsection{Anastomoses et symbioses}

Une racine ne reste jamais seule. Pour vivre, elle a besoin de se démultiplier, de se ramifier en radicelles qui forment un système racinaire étendu et plus ou moins enchevêtré. Il arrive aussi que les racines de deux plantes fusionnent entre elles, formant des anastomoses qui leur permettent d'échanger matières et signaux. Le plasticien Hubert Duprat ${ }^{33}$ a utilisé cette figure dans son œuvre $\grave{A}$

\footnotetext{
32 Dov Bernard Hercenberg, "Invisibilité de l'être-ensemble. Des racines et d'un arbre à cernes ", Centre Sèvres / Archives de Philosophie, Tome 77, 2014/3, p. 512. Article disponible en ligne à l'adresse : https://www.cairn.info/revue-archives-dephilosophie-2014-3-page-507.htm

${ }^{33}$ Hubert Duprat : artiste français né en 1957 à Nérac (Lot-et-Garonne), vit dans le sud de la France. Son œuvre est à la croisée de l'art et de la science. L'histoire de l'artisanat et de l'artefact, le geste et le rapport à l'outil sont ses champs d'intérêt récurrents. En 1983, il propose deux types de travaux bien différents : $1^{\circ}$ des larves d'insectes aquatiques (trichoptères), qui ont pour (C) 2020 ISTE OpenScience - Published by ISTE Ltd. London, UK - openscience.fr 
la fois, la racine et le fruit (Fig. 8), un entrelacs de deux branches d'arbre ramifiées, serties de fines plaquettes d'os polis évoquant l'ivoire, et clouées par des pointes. La matière principale de cette sculpture (bois) est doublement occultée : par les lamelles osseuses qui la recouvrent, et par son titre même, lié à la racine et au fruit. Ce sont bien des branches, mais qui ressemblent à deux racines anastomosées. Le fruit annoncé est absent, sauf à le rapporter à l'œuvre elle-même...

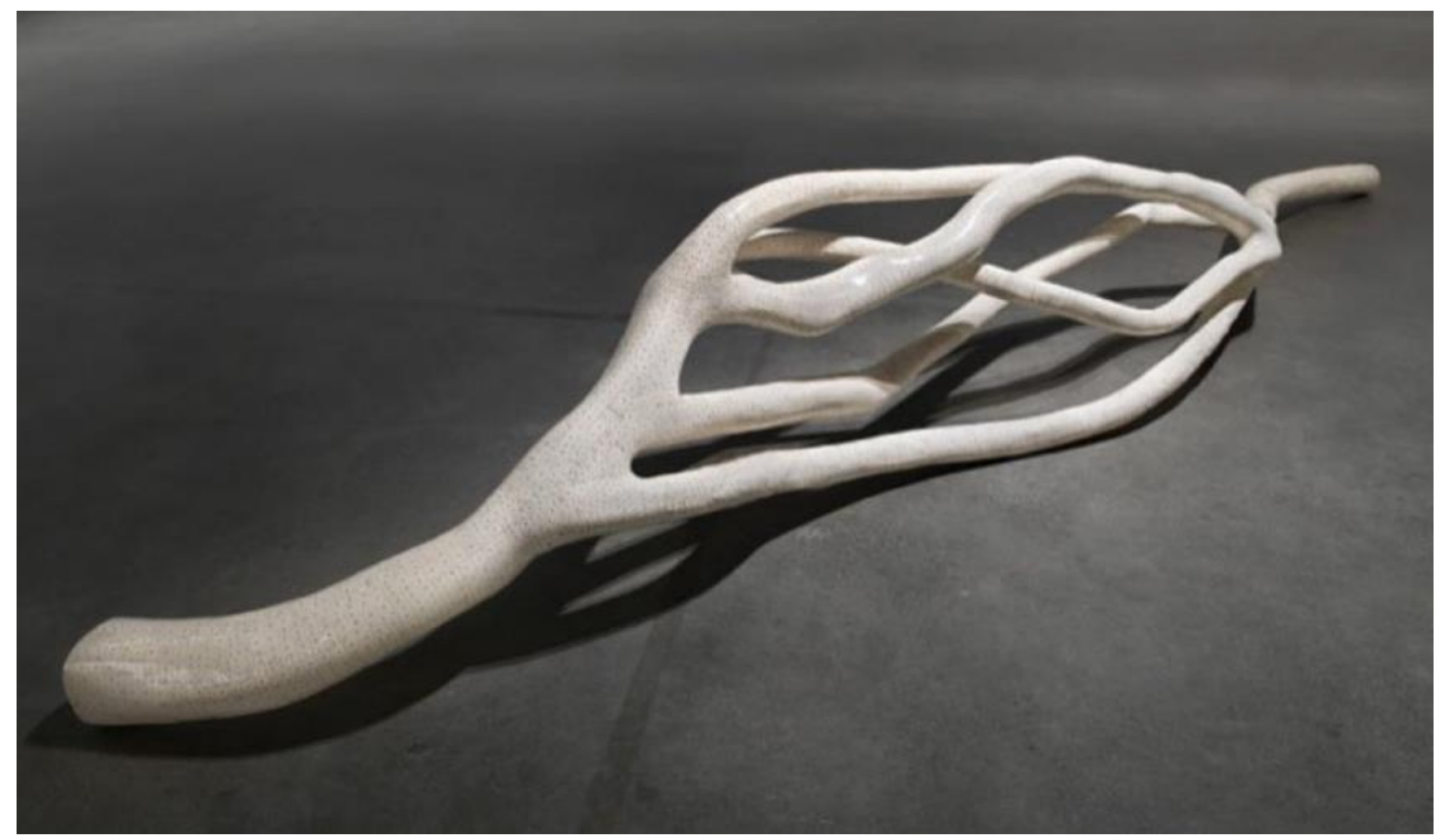

Figure 8. Hubert Duprat, À la fois, la racine et le fruit, 1997-1998, bois et plaquettes d'os de bovins, $380 x$ $60 \times 60 \mathrm{~cm}$. Collection Fondation Cartier pour l'art contemporain, Paris, (C) Hubert Duprat, ADAGP, Paris, 2020

Les racines aiment à se mélanger, non seulement entre elles mais avec toutes sortes d'éléments de leur entourage, à commencer par la terre. Racines et terre sont d'ailleurs si inséparables que déraciner totalement une plante s'avère impossible! Plus précisément, certaines racines ${ }^{34}$ établissent des associations symbiotiques avec divers êtres vivants - microorganismes, bactéries et champignons. Une des symbioses les mieux connues a lieu à l'intérieur des nodules racinaires de légumineuses, qui hébergent des bactéries rhizobium ; celles-ci peuvent fixer une grande quantité d'azote à partir de l'air, contribuant ainsi à la croissance des plantes. Encore plus importantes car répandues dans l'ensemble du monde végétal, les mycorhizes (du grec mûkes/champignon et rhiza/racine) ont été décrites pour la première fois en 1885 par le botaniste allemand Albert Frank mais leur rôle n'est connu que depuis les années 1950. Ce sont des symbioses mutualistes entre

particularité d'utiliser des matériaux de leur environnement pour se construire un étui protecteur; en plaçant dans leur aquarium des perles, des paillettes d'or et des pierres précieuses, il les contraint à fabriquer de l'orfèvrerie. $2^{\circ}$ des photographies qui reproduisent la projection d'une portion de l'espace extérieur sur un mur de l'atelier de l'artiste, suivant la méthode de la camera obscura. Au cours des années suivantes, il entame une réflexion sur la fragmentation et la recomposition. Le maître mot de son œuvre est la marqueterie, par laquelle il fait se répondre des matières animales, végétales et minérales. Du 18 septembre 2020 au 10 janvier 2021, le Musée d'Art moderne de Paris lui consacre une première exposition rétrospective.

34 II y a lieu de distinguer entre les " racines de structure » et les " racines fines " (voir Francis Hallé, " Des feuilles souterraines ? ", dans Alliage $n^{\circ} 64$, mars 2009). Seules les racines fines sont concernées par la symbiose mycorhizienne, qui existe dans $90 \%$ des plantes. 
racines et champignons microscopiques du sol. Dans ces échanges de bons procédés (dans lesquels on se gardera toutefois de voir de l'altruisme ou de la bienveillance), les racines fournissent aux champignons le carbone dont ils ont besoin, et les champignons apportent à la racine de l'eau, des sels minéraux (potassium, phosphore) et de l'azote.

Cette hybridation particulièrement intime - l'un des deux organismes pénétrant dans les tissus de l'autre, voire même dans ses cellules - concerne l'immense majorité des plantes actuelles, auxquelles elle apporte encore bien d'autres bénéfices. Grâce au réseau très étendu de leurs filaments mycéliens microscopiques (hyphes), les champignons multiplient par cent ou mille la zone explorée par les racines; un seul champignon peut s'associer aux racines fines de nombreuses plantes d'espèces différentes, et un seul gramme de sol forestier peut contenir près de 200 mètres d'hyphes ! La mycorhize densifie également le système racinaire, ce qui accélère la croissance végétale et enrichit la floraison et la fructification. Enfin les champignons renforcent les défenses des plantes contre les attaques de pathogènes, parasites ou ravageurs, et leur permettent de mieux résister aux stress dus à la pollution et au manque d'eau. Ce maillage interspécifique, que l'on a comparé au réseau Internet, a encore d'autres effets surprenants : un même champignon mycorhizien est capable par exemple d'interconnecter les systèmes racinaires de deux plantes en transférant des molécules carbonées de l'une à l'autre; et dans les sous-bois, les plantes qui manquent de lumière, peuvent puiser leur nourriture chez les arbres environnants qui leur font de l'ombre.

Toutes ces interconnexions ont inspiré le plasticien Fabrice Hybert ${ }^{35}$, frappé depuis quelques années par tout ce que les arbres mettent en œuvre en termes de communications racinaires. Son œuvre Paysage de mesures (Fig. 9), qui appartient à sa série de «peintures rhizomatiques », est une sorte d'état des lieux aussi poétique que scientifique, une riche interprétation des contacts « intelligents » entre racines issues d'arbres différents - contacts tactiles entre les extrémités de racines multicolores paraissant se donner la main (au premier plan); contacts bioélectriques représentés par un tracé ondulatoire entre les bases des deux troncs les plus centraux, chaque sommet du tracé étant lui-même relié à l'arbre de l'arrière-plan. Cette interconnexion généralisée des systèmes racinaires est redoublée par celle des troncs, chaque tronc d'un même groupe d'arbres fusionnant avec les autres par sa base. D'autres ondes, venues du ciel, se ramifient comme des branches ou des racines pour atteindre les divers arbres jusqu'en bas. Ce paysage conceptuel est hors temps, les arbres de gauche, peints en gris, seraient plutôt nocturnes alors que ceux de droite, verts, assument seuls la synthèse chlorophyllienne, tout en étant dépourvus de feuilles.

\footnotetext{
${ }^{35}$ Fabrice Hybert ou Hyber : plasticien français né en 1961 à Luçon (Vendée), vit et travaille à Paris et en Vendée. Artiste prolifique, Fabrice Hyber se déplace entre la peinture, le dessin, la sculpture, l'installation, la performance, la photographie et la vidéo. Sensible aux sciences biologiques, astronomiques, mathématiques et physiques, il traduit leurs questionnements dans ses œuvres. Ses Peintures homéopathiques montrent ainsi tout ensemble le sujet traité (paysage, corps ou objet) et les étapes du processus créatif (calculs, documents de recherches, croquis, ou états d'esprit de l'artiste), qui font partie intégrante de l'œuvre. Nombreuses expositions personnelles en Europe, aux États-Unis et en Asie. Lion d'or à la Biennale de Venise en 1997, Officier dans l'Ordre des Arts et des Lettres depuis janvier 2012, Fabrice Hybert est élu à l'Académie des beaux-arts en 2018. Une dizaine d'ouvrages lui sont consacrés.
} 


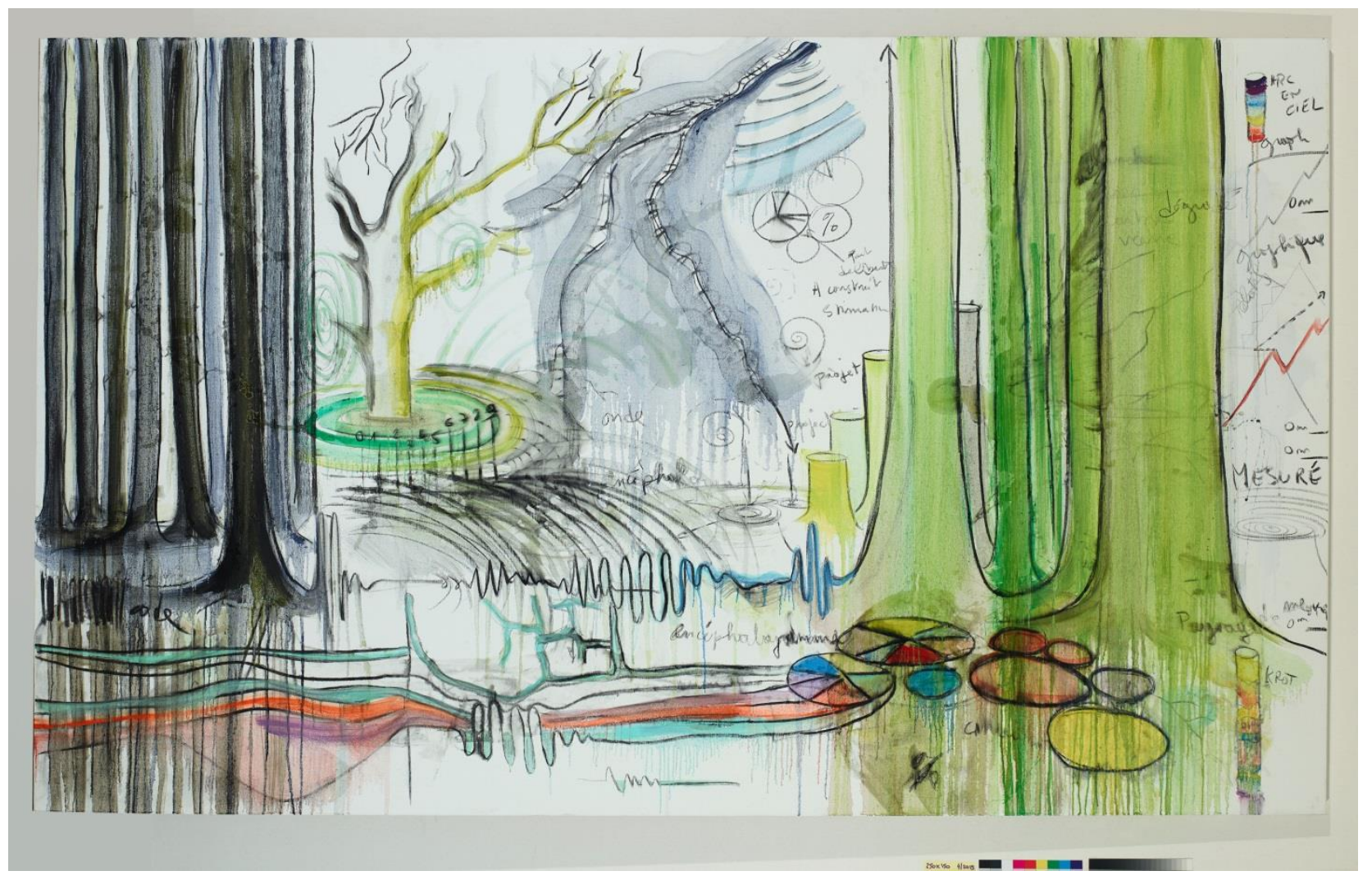

Figure 9. Fabrice Hyber, Paysage de mesures, 2019. Huile et fusain sur toile, $150 \times 250 \mathrm{~cm}$. Collection de l'artiste (c) Fabrice Hyber

\section{Devenir racine}

\subsection{Rêves fusionnels}

Avant même que les botanistes ne voient dans les racines les plus formidables des messagers souterrains, les poètes et les artistes les ont rêvées de diverses manières, voire se sont rêvés en elles. Dans les années 1830, l'écrivain Maurice de Guérin s'imagine ainsi fusionner avec les vies minuscules qui colonisent les racines : «Je voudrais être l'insecte qui se loge et vit dans la radicule, je me placerais à la dernière pointe des racines et je contemplerais l'action puissante des pores qui aspirent la vie; je regarderais la vie passer du sein de la molécule féconde dans les pores qui, comme autant de branches, l'éveillent et l'attirent par des appels mélodieux. Je serais témoin de l'amour ineffable avec lequel elle se précipite vers l'être qui l'invoque et de la joie de l'être. J'assisterais à leurs embrassements ${ }^{36}$. » Dans cet extrait, l'exaltation romantique propre à cet auteur, va de pair avec une exactitude botanique remarquable pour son époque.

Dans une veine similaire, citons un nouvel extrait du Dialogue de l'Arbre de Paul Valéry, dans lequel le poète et philosophe Lucrèce se voit fusionner, par-delà la racine, avec la plante tout entière : «... Je voulais te parler du sentiment que j'ai, parfois, d'être moi-même Plante, une Plante, qui pense, mais ne distingue pas ses puissances diverses, sa forme de ses forces, et son port de son lieu. Forces, formes, grandeur, et volume, et durée ne sont qu'un même fleuve d'existence, un flux dont la liqueur expire en solide très dur, tandis que le vouloir obscur de la croissance s'élève, éclate, et veut redevenir vouloir sous l'espèce innombrable et légère des graines. Et je me sens vivant l'entreprise inouïe du Type de la Plante, envahissant l'espace, improvisant un rêve de ramure, plongeant en pleine fange et s'enivrant des sels de la terre, tandis que dans l'air libre, elle ouvre par degrés aux largesses du ciel des milliers verts de lèvres... Autant elle s'enfonce, autant s'élève-t-elle

${ }^{36}$ Maurice de Guérin, Le cahier vert, Paris, éd. Le Divan, I, 1832-1835, p. 246 
: elle enchaîne l'informe, elle attaque le vide ; elle lutte pour tout changer en elle-même, et c'est là son Idée !... Ô Tityre, il me semble participer de tout mon être à cette méditation puissante, et agissante, et rigoureusement suivie dans son dessein, que m'ordonne la Plante ${ }^{37} \ldots$ » Par cette merveilleuse vision onirique, le poète saisit la totalité de la plante dans son élan vital indivis ; avec elle et en elle, il participe, de façon aussi rigoureuse que mystique, aux mouvements aériens et souterrains du grand «fleuve de la vie». C'est enfin un rêve de fusion au-delà des formes, que nous offre François Solesmes, une identification aux conditions obscures de la vie racinaire : «Pénétrer le dédale. Se faire nuit enclose et sève aveugle que l'on exhorte et fourvoie de fourche en fourche ${ }^{38}$. »

\subsection{Pensées radicales}

De nombreux auteurs - philosophes, écrivains, plasticiens, botanistes de toutes les époques - ont usé de l'analogie entre l'homme et la plante: l'arbre en particulier, figure emblématique de l'homme debout. Par ailleurs, l'altérité de la vie végétale au regard de la vie animale (humaine ou non) fait l'objet d'un large consensus. Nous voici donc contraints de penser aux plantes en termes de similitude et d'opposition par rapport aux humains que nous sommes... Cette situation inconfortable constitue le point de départ des débats contemporains sur le statut philosophique des plantes en tant qu'êtres vivants.

Tentons brièvement de cerner plus précisément l'altérité supposée des végétaux et ses conséquences sur les regards et les comportements humains envers le monde végétal. L'autotrophie qui résulte de la synthèse chlorophyllienne, est sans conteste la spécificité majeure des plantes, et leur altérité à cet égard est en effet radicale. Concernant l'individualité, la sensibilité et l'intelligence, plusieurs thèses s'affrontent. Notre propos est simplement d'en indiquer les grandes lignes et les principaux enjeux, dans la mesure où elles retentissent sur les diverses manières de concevoir les racines.

Pour la plupart des auteurs, la plante n'est pas un individu : elle germe mais ne nait pas, et sa mort n'est jamais due à une nécessité interne; elle est dépourvue de centre intégrateur tel que le système nerveux central des animaux, responsable de la vie vécue par un organisme ; la plante «ne sait pas, elle n'utilise rien, elle n'a ni besoins, ni projets, ni buts. Nous parlons un langage d'animaux qui se prête mal à la relation d'une vérité végétale ${ }^{39}$. "S'il faut donc renoncer à dire scientifiquement une "vérité végétale ", celle-ci peut être approchée par d'autres voies - une science poétisée, comme sous la plume du botaniste Jacques Tassin : « Tout en l'arbre est ouvert et offert au monde. [...] L'arbre concilie les contraires, transcende les frontières, multiplie l'intersubjectivité en connectant entre eux les êtres vivants ${ }^{40} . »$; la littérature, comme dans cet extrait de L'arbre-monde de Richard Powers: «Les arbres de Patricia sont bien plus sociables qu'elle-même n'aurait pu le soupçonner. Il n'y a pas d'individus. Il n'y a même pas d'espèces séparées. Tout ce qui est dans la forêt est la forêt. La concurrence n'est pas séparable des multiples saveurs de la coopération ${ }^{41}$.»; la philosophie, quand Raymond Ruyer se demande, dans une étude consacrée à l'individualité ${ }^{42}$, s'il est possible d'être «plus ou moins un individu », et conclut qu'en

\footnotetext{
${ }^{37}$ Paul Valéry, op. cit., p. 177-178

${ }^{38}$ François Solesmes, op. cit., p. 27

${ }^{39}$ Francis Hallé, Éloge de la plante. Pour une nouvelle biologie, Paris, Seuil, 1999, p. 324

40 Jacques Tassin, Penser comme un arbre, Paris, Odile Jacob Poches, 2018, p. 34

${ }^{41}$ Richard Powers, L'arbre-monde, traduit par Serge Chauvin, Paris, Cherche Midi 10/18, 2018, p. 221

${ }^{42}$ Raymond Ruyer, "L'individualité », Revue de métaphysique et de morale, n 47, vol. 3, 1940, p. 286 
biologie, «l'individualité est un caractère flottant » et que les organismes multicellulaires, dans leur diversité, montrent « tous les degrés possibles d'individualité ».

Au début des années 2000, de nouvelles recherches, portant sur la communication végétale, postulent l'existence d'une sensibilité multiple se déployant en authentique « intelligence végétale ». Le professeur Stefano Mancuso, de l'université de Florence, signe en 2006, avec cinq collaborateurs, un article destiné à fonder une nouvelle discipline scientifique, la «neurobiologie végétale ${ }^{43}$. De nombreux ouvrages et films ont émergé récemment autour de ces questions, et sont souvent devenus des best-sellers (pour le meilleur et pour le pire); dus aux biologistes eux-mêmes ${ }^{44}$ mais aussi à un ingénieur forestier ${ }^{45}$, à des écrivains ${ }^{46}$, à des philosophes ${ }^{47}$ et des réalisateurs ${ }^{48}$ (liste indicative), ils ont fortement contribué au débat public, toujours vigoureux à l'heure actuelle.

Pour nous en tenir aux racines, l'analogie fonctionnelle entre la racine et le cerveau a une longue histoire : au Moyen Âge, pour Guillaume de Conches, la tête de l'homme « est comme sa racine »; au XIX ${ }^{\text {ème }}$ siècle, Darwin le dira lui aussi, avec cette précaution de langage : «Il est à peine exagéré de dire que la pointe radiculaire [...] agit comme le cerveau d'un animal inférieur. » Notons cependant qu'à la même époque, Lorenz Oken attribue la fonction cérébrale à la fleur et non à la racine : «La fleur est le cerveau des plantes, le correspondant de la lumière, qui reste ici sur le plan du sexe. On peut dire que ce qui est sexe dans la plante est pour l'animal cerveau, ou que le cerveau est le sexe de l'animal ${ }^{49}$. » De nos jours, les promoteurs de l'intelligence végétale ont renchéri sur l'analogie racine-cerveau, qu'ils ont transformée en réelle équivalence - au prix d'une réduction de la polysémie originelle du terme intelligence (celle-ci étant alors définie uniquement comme la capacité des êtres à communiquer entre eux et à s'adapter à leur environnement), et au risque d'utiliser à propos des plantes un langage truffé de termes psychologisants (instinct maternel, solidarité, émotions, conscience, etc.) qui suggèrent une intériorité peu adaptée aux organismes végétaux. Notons cependant l'approche subtile de Jacques Tassin : «S'il faut leur reconnaître une intelligence, c'est d'abord dans leur capacité, en se décentrant, à composer en permanence avec le monde $^{50}$ » et la position audacieuse d'Emanuele Coccia, qui, tout en adhérant à la thèse de «l'intelligence végétale», vise plus spécifiquement à «élargir le sens des notions de savoir et de pensée, dans une direction opposée à celle de l'aristotélisme. Non pas faire de l'intellect un organe séparé, mais bien de le faire coïncider avec la matière ${ }^{51}$. »

Tout comme le monde végétal dans son ensemble, les racines, qui inspirent aux humains tant de rêves et de créations, suscitent aussi en eux des pensées articulées voire des théories quant à leurs

\footnotetext{
2020.

${ }^{44}$ Jacques Tassin, À quoi pensent les plantes ? Paris, Odile Jacob, 2016, p. 120

${ }^{45}$ Peter Wohlleben, La vie secrète des arbres, Paris, Les Arènes, 2017

${ }^{46}$ Richard Powers, op. cit.

${ }^{47}$ Florence Burgat, op. cit. ; Emanuele Coccia, op. cit.

${ }^{48}$ L'intelligence des arbres, documentaire de Julia Dordel et Guido Tölke, 2017

${ }^{49}$ Lorenz Oken, Lehrbuch der Philosophie, $3^{\text {ème }}$ éd., Zürich, Friedrich Schultheiss, 1843, p. 218

50 Jacques Tassin, op. cit., p. 43

${ }^{51}$ Emanuele Coccia, op. cit., p. 134
}

43 Anthony Trewavas, Plant Behaviour and Intelligence, Oxford University Press, 2014 : Stefano Mancuso et Alessandra Viola, L'Intelligence des plantes, Paris, Albin Michel, 2018. Voir aussi Jacques Tassin, Penser comme un arbre, Paris, Odile Jacob, Essais, 
fonctions biologiques et leur statut philosophique. Au-delà des controverses dont nous avons brièvement fait état, il est incontestable à l'heure actuelle que la plante n'est pas cantonnée à la vie passive dont on l'a longtemps créditée. Par son propre enracinement, elle s'unit à la terre dont elle devient inséparable : ses racines, qui ne cessent de croître et de se ramifier, voire de se réitérer, étendent son terrain d'action et déjouent son apparente immobilité.

Pour Emanuele Coccia, cette «immersion est une relation plus profonde que l'action et la conscience - elle est en deçà de la praxis comme de la pensée. [...] En cela, les plantes nous donnent à voir la forme la plus radicale de l'être-au-monde. »Une radicalité qui agit sur le monde sans «nul besoin de fabriquer un objet différent de soi (en déversant de la matière en dehors de sa peau) ni de percevoir, de reconnaître, de viser directement et consciemment une portion du monde et vouloir le changer ${ }^{52}$. » En bonne logique, il n'y aurait donc pas plus radical que les racines, et leur radicalité renverrait à la profondeur même de l'exister, autrement dit à ce qu'on pourrait se hasarder à appeler une vérité racinaire. Et pourtant, c'est en vain que l'on chercherait dans un dictionnaire, à la rubrique «Botanique », le terme radicalité ; en s'appropriant le sens fondamental et originaire de radical, les sciences humaines en ont dépossédé les racines... Leurs éléments peuvent bien être radicaux, à condition d'oublier leur radicalité même ${ }^{53}$ ! Quant à 1'adjectif racinaire, il n'appelle aucune «racinarité »... Autre étrangeté lexicographique, dans la famille des termes apparentés à racine, le verbe radicaliser semble en être encore plus éloigné que radicalité : les racines végétales ne se radicalisent pas plus qu'elles ne sont susceptibles d'être radicalisées... On voit ici comment un champ lexical peut migrer en se détournant de son domaine originel.

Dans son texte «Images terre à terre », Georges Didi-Huberman tente pourtant de définir ce que pourrait être une radicalité des racines : «ce serait justement qu'elles sont là et non pas au-delà, juste sous nos pas, tout autour de nous, et non pas dans le ciel des idées, et pas plus au fond archétypique de quelque source "véritable" ou antiquité "inaccessible". De l'inaccessible, il y en a, bien sûr. Les racines, nous ne pouvons jamais les voir tout à fait, les saisir - maîtriser leur logique ou les tenir entièrement entre nos mains. Elles sont faites de latences, d'oublis, de destructions, d'intermittences ${ }^{54} \ldots \gg$ On retrouve bien dans cette vision de la radicalité racinaire, la vie ancrée ici et maintenant, mais du fait de sa discontinuité, cette vie est vouée à nous échapper partiellement. Poursuivant sa réflexion, Didi-Huberman revient sur le sens du verbe radicaliser et s'interroge : «Radicaliser, est-ce vraiment aller à la racine des choses? Ma petite promenade dans la forêt fait à cela deux objections. Premièrement, on ne va pas "à la racine" parce que la racine n'existe pas : il n'y a que des racines, une quantité nécessairement indéfinie, pullulante et incalculable, vivace et monstrueuse quelquefois, de racines. Une seule racine, à supposer que l'on puisse l'isoler, produit le plus souvent d'innombrables bifurcations radiculaires. Freud avait un mot pour cela, le mot "surdétermination". Sans compter le "rhizome" selon Deleuze et Guattari. J'ai l'impression, ici, que si je tirais très fort un seul brin, un seul bout de ce réseau, c'est toute la forêt, peut-être même toute la montagne qui sortiraient d'elles-mêmes ${ }^{55}$. »

Toujours est-il que l'origine végétale de radicaliser est incontestable, ce qui nous invite à concevoir la pensée radicale comme étant réellement à l'image des racines - enracinée mais mouvante, audacieuse et connectée à d'autres pensées. Didi-Huberman, encore : «Parce que les

\footnotetext{
52 Ibid., p. 56

${ }^{53}$ En botanique, « radical » se dit d'un élément lié aux racines de la plante, par exemple des feuilles qui naissent au collet de la plante. Mais la « radicalité » botanique est introuvable...

${ }^{54}$ Georges Didi-Huberman, « Images terre à terre », op. cit.

55 Ibid.
} 
racines ne font pas que fixer l'arbre à la terre mais lui assurent aussi quelque chose comme un mouvement migratoire qui le fait "toucher" d'autres arbres - selon un processus dit d'anastomose -, la pensée radicale serait donc une pensée capable de migrer hors d'elle-même, une pensée capable de mettre en question ses propres fondements [...] Être radical, ne serait-ce pas, alors, être capable de se déraciner soi-même, de faire migrer son propre terrain de pensée, de s'inventer des sortes de "radicaux libres"? Être radical, ne serait-ce pas, tout simplement, savoir changer de radicall ${ }^{56}$ ?" Les botanistes contemporains nous avaient déjà invités à assumer des «vertus végétales » telles que l'humilité, la patience, la coopération... Georges Didi-Huberman est à la fois plus prudent et plus novateur, comme s'il avait fait sienne non seulement la part incertaine de la vie racinaire mais aussi ses percées libératrices. 